\title{
Abordagem em Realidade Aumentada para Momentos de Aprendizagem Autodirigida
}

\author{
Helen de Freitas Santos ${ }^{1}$, Gabriel de Freitas Santos Sábio ${ }^{1}$, Wanderley Lopes de \\ Souza $^{2}$ \\ ${ }^{1}$ Departamento de Informática - Instituto Federal de Educação Ciência e Tecnologia de \\ São Paulo (IFSP), Campus Birigui \\ Rua Pedro Cavalo 709 - CEP 16.201-407 - Birigui - SP - Brasil \\ ${ }^{2}$ Departmento de Computação - Universidade Federal de São Carlos (UFSCar) \\ Rodovia Washington Luís, Km 235 - CEP 13.565-905 - São Carlos - SP - Brasil \\ helen@ifsp.edu.br, gabriefreitas@gmail.com, desouza@ufscar.br
}

\begin{abstract}
This paper shows the ABORA, an Augmented Reality Approach to Self Directed Learning Moments in Courses Based on Active Learning Methodologies. In order to evaluate the ABORA, a software technology must be developed. The software technology architecture was proposed, it is also showed here and part of this architecture named Educational and Academic Management System was developed. This study aims to gather education, innovative technologies and actors in the teaching-learning process in order to make the process activities more significant and interesting.
\end{abstract}

Resumo. Este artigo apresenta a ABORA, uma Abordagem em Realidade Aumentada para Momentos de Aprendizagem Autodirigida em Cursos Baseados em Metodologias Ativas de Aprendizagem. A fim de avaliar a ABORA, uma tecnologia de software deve ser desenvolvida. A arquitetura dessa tecnologia de software foi proposta, é aqui apresentada e parte dessa arquitetura, o Sistema de Gestão Pedagógica e Acadêmica, foi desenvolvida. Com este estudo busca-se aproximar educação, tecnologias inovadoras e atores do processo ensino-aprendizagem com o objetivo de tornar as atividades do processo mais significativas e interessantes.

\section{Introdução}

Desde os primórdios, a Educação tem se configurado pela transferência do conhecimento do docente para o estudante, com este recebendo-o sem a devida crítica e reflexão e não se apoderando do conhecimento. Assim, como consequências encontramse: 1) a aprendizagem do estudante, objetivo principal do ensino, não é alcançada, 2) constantes questionamentos quanto à metodologia tradicional de ensino e 3) surgimento de novas metodologias de ensino, como as Metodologias Ativas de Aprendizagem (MAA). MAA empregam a problematização como estratégia de ensino-aprendizagem, onde o estudante constrói seu próprio conhecimento a partir de problemas que lhe são apresentados, tornando-se assim um ser reflexivo e crítico, e têm períodos reservados, denominados espaços de aprendizagem autodirigida (AAD), nos quais pode dedicar-se à busca de novas informações [Ribeiro 200?]. 
Por outro lado, com o advento das novas Tecnologias da Informação e Comunicação (TICs), a interação comunicativa altera o modo de ser e de agir das pessoas, fazendo-se presente em todos os setores da vida social e profissional, na rotina diária do homem, no interior de sua casa, nas salas de aula, etc.

$\mathrm{Na}$ Educação, as TICs têm sido cada vez mais utilizadas nas últimas décadas e são capazes de expressar representações da realidade ou da imaginação, sendo imperativo explorar, dentro das mais diversas áreas do conhecimento, as potencialidades oferecidas pelas TIC's. Nesta gama de possibilidades providas pelo avanço dos recursos computacionais destaca-se a realidade aumentada (RA), vislumbrada como nova forma de visualização da informação em aplicações computacionais. Segundo Braga (2001), o avanço tecnológico vem possibilitando aos computadores pessoais tornarem-se cada vez mais rápidos, fazendo com que a RA deixe de ser objeto de estudo somente dos grandes centros de pesquisa, passando a ser utilizada por usuários comuns, no ambiente escolar, fomentando e fortalecendo abordagens inovadoras e, consequentemente ressignificando aspectos voltados às questões relativas ao ensino-aprendizagem. Neste âmbito, RA abarca diversas metodologias, motiva o ambiente ensino-aprendizagem, oferece grande poder ilustrativo e de interação e pode ser muito útil nos momentos de AAD. Neste sentido, buscando aliar tecnologias inovadoras à educação, este artigo apresenta uma Abordagem em RA (ABORA) para momentos de AAD e uma arquitetura de software para avaliar tal abordagem.

\section{Realidade Aumentada: Uma Tecnologia Inovadora}

RA é um termo que descreve as tecnologias que permitem misturar, em tempo real, os conteúdos gerados por computador e o mundo real [Haller, apud Owen 2011], proporcionando novos usos de aplicação de RA que permitem convergir as áreas de educação e entretenimento, criando assim novas oportunidades para apoiar o ensinoaprendizagem em contextos formais e informais [Salmi, apud OWEN 2011].

Buscando a origem do termo "Augmented Reality" na internet, diversos sites informam que o termo foi cunhado pelo pesquisador Tom Caudell, em 1990, quando ele e um colega, trabalhadores da Boeing, foram convidados a criar uma alternativa aos dispositivos e diagramas caros, usados para guiar trabalhadores no chão de fábrica. Hoje, passados 26 anos, a RA ainda não está completamente difundida e popularizada, dando origem a uma questão: RA é uma tecnologia inovadora? Para respondê-la, realizou-se três estudos para analisar a capacidade inovadora da RA: os resultados do Google Trends, em busca do termo "Augmented Reality"; busca pela palavra "realidade" nos artigos publicados no Simpósio Brasileiro de Informática na Educação (SBIE) entre 2001 e 2015 e relatório elaborado pelo New Media Consortium (NMC), uma comunidade internacional formada por especialistas em tecnologias educacionais, cujo papel é ajudar seus membros a impulsionar a inovação em seus campi, através do NMC Horizon Project [NMC 2016].

Com o primeiro estudo, através do Google Trends, uma ferramenta do Google que mostra a popularidade dos termos ao longo dos anos, observa-se que o termo "Augmented Reality" inicia um crescimento após 2009, atinge o pico logo após 2012 e ultimamente está numa gangorra (figura 1). Quanto às publicações no SBIE ao longo de 15 anos, os artigos contendo a palavra "realidade" no seu título, envolvendo realidades 
V Congresso Brasileiro de Informática na Educação (CBIE 2016)

Anais do XXVII Simpósio Brasileiro de Informática na Educação (SBIE 2016)

virtual (RV) e aumentada, representam, em média, apenas 1,20\% do total de publicações. Duas edições, nesse período, não têm nenhuma publicação: 2001 e 2004. Após 2009 tem início um crescimento na publicação de estudos de RA (figura 2), o que coincide com o resultado apresentado pelo Google Trends. A terceira pesquisa diz respeito ao NMC. Na série Horizon Report (HR) para o ensino superior (EES) observase que desde 2012 o NMC sinaliza RA como tecnologia emergente. No entanto, nos relatórios de 2012 a 2016 [Johnson 2012; 2013; 2014; 2015; 2016] observa-se que a previsão para a concretização dessa tendência oscila (tabela 1).

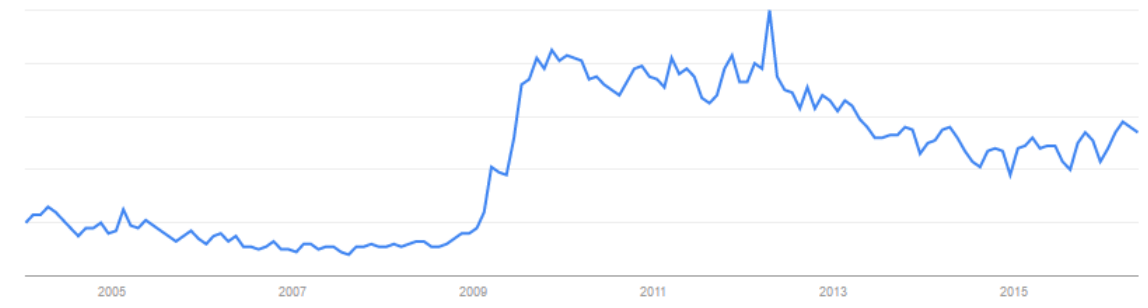

Figura 1. Popularidade do Termo "Augmented Reality" ao Longo dos Anos através da Ferramenta Google Trends

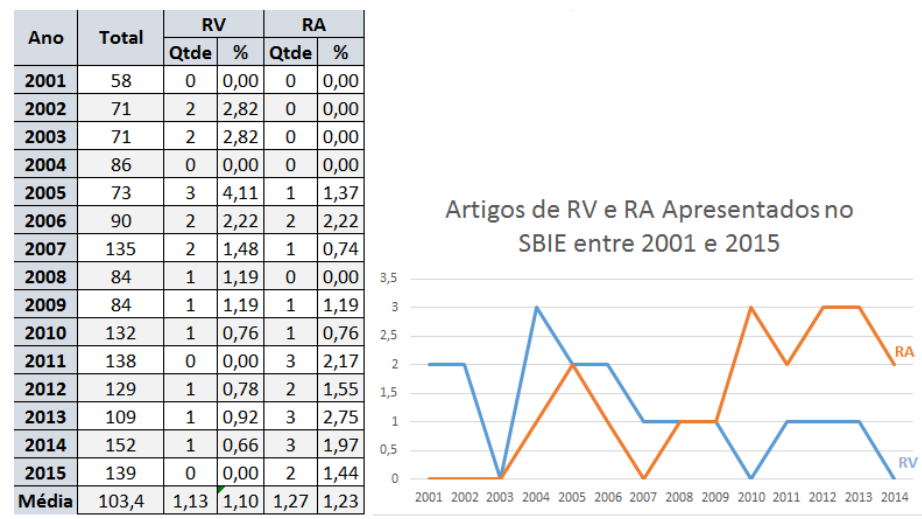

Figura 2. Resultado da Pesquisa pela Palavra "Realidade" nas Publicações no SBIE entre 2001 e 2015

Tabela 1. Tecnologias Emergentes Sugeridas nos Relatórios do NMC de 2012 a 2016

\begin{tabular}{|c|c|c|c|}
\hline Ano Publicação & Tecnologia Educacional & Prazo & Previsão \\
\hline 2012 & Computação Baseada em Gestos & Longo Prazo (4 a 5 anos) & 2017 \\
\hline 2013 & Impressoras 3D, Tecnologias Trajáveis & Longo Prazo (4 a 5 anos) & 2018 \\
\hline 2014 & Uso de Abordagens Ágeis no Ensino & Longo Prazo (acima 5 anos) & 2019 \\
\cline { 2 - 4 } & Impressão em 3D & Médio Prazo (2 a 3 anos) & 2017 \\
\hline 2015 & Tecnologias Trajáveis & Médio Prazo (2 a 3 anos) & 2018 \\
\hline 2016 & Realidade Aumentada & Médio Prazo (2 a 3 anos) & 2019 \\
\hline
\end{tabular}

Após analisar os estudos aqui apresentados conclui-se que RA é realmente uma tecnologia inovadora e a oscilação quanto ao seu uso deve-se a alguns fatores: 1) não é tecnologia de fácil implementação; 2) não é tecnologia amplamente disseminada quanto à prática de sua implementação; 3) seu uso mais comum está no setor de consumo e entretenimento e não em aplicações voltadas para a educação; 4) surgimento recente de tecnologias de hardware, como os óculos Microsoft HoloLens e óculos Rift. Tal 
V Congresso Brasileiro de Informática na Educação (CBIE 2016)

Anais do XXVII Simpósio Brasileiro de Informática na Educação (SBIE 2016)

conclusão também confirma-se com o relatório de 2016 do NMC por meio do surgimento do termo RA e em sua previsão de uso para 2019. Com as indicações apresentadas pelos membros do NMC, aliar a tecnologias de RA à educação parece um caminho a ser explorado para oferecer uma abordagem e um ferramental aos profissionais da educação e aos estudantes.

\section{Práticas Pedagógicas Inovadoras}

MAA é uma concepção educativa que estimula a crítica e reflexão no processo de ensino-aprendizagem, que eleva o nível de participação do estudante, indo em direção contrária aos métodos e técnicas que enfatizam a transmissão do conhecimento. $\mathrm{O}$ educador participa ativamente do processo em situações que fazem do estudante um ser crítico da realidade. Tais metodologias empregam a problematização como estratégia de ensino-aprendizagem, onde o estudante constrói seu próprio conhecimento a partir de problemas que lhe são apresentados, tornando-se um ser reflexivo e crítico.

As principais metodologias ativas usadas atualmente são Problem Based Learning (PBL), em Português Aprendizagem Baseada em Problemas, Metodologia da Problematização (MP) e Aprendizagem Baseada em Projetos (ABP). Quando PBL é usada, a construção do conhecimento ocorre durante a interação entre os atores do processo por meio de sucessivas assimilações. Essa metodologia é centrada no estudante e se desenvolve em pequenos grupos, sendo um processo ativo, cooperativo, integrado, interdisciplinar e orientado à aprendizagem de adultos [Santana 2009]. Uma atividade proposta em cursos que utilizam MAA é AAD, que também deve ser exercitada em cursos que utilizam metodologias tradicionais. Os momentos de AAD são espaços em que estudantes dedicam seu tempo para estudo e pesquisa, com o intuito de se apoderar do conhecimento, e ocorrem após a problematização, quando o aluno se dedica à reflexão [Ribeiro 200?]. No Brasil, cursos da Universidade Federal de São Carlos (UFSCar) e do Instituto Sírio Libanês de Ensino e Pesquisa (IEP) empregam MAA. Tais cursos são referenciados aqui como Cursos Baseados em MAA (CBMAA) e se "apoiam nas experiências e na fundamentação teórica da aprendizagem baseada em problemas, utilizando a espiral construtivista como referencial" [Ribeiro 200?, Tempski 2014], a qual é formada por seis movimentos, percorridos em sentido anti-horário: identificando o problema, formulando explicações, elaborando questões, buscando novas informações, construindo novos significados e avaliando o processo.

A inovação das práticas pedagógicas e a inserção das TICs nessas práticas ainda não está consolidada e muito tem se discutido a esse respeito.

A UNESCO percebeu essa tendência e a expressou em uma série denominada Padrões de Competência em TICs para Professores, em três volumes: Diretrizes de Implementação, Marco Político e Módulos de Padrão de Competência. Compartilhamos da mesma ideia do Diretor do Setor de Comunicação [Unesco 2009]:

\footnotetext{
“As práticas educacionais tradicionais já não oferecem aos futuros professores todas as habilidades necessárias para capacitar os alunos a sobreviverem no atual mercado de trabalho. Tanto os programas de desenvolvimento de profissionais na ativa e os programas de preparação dos futuros professores devem oferecer experiências adequadas em tecnologia em todas as fases do treinamento”.

E mais...
} 
V Congresso Brasileiro de Informática na Educação (CBIE 2016)

Anais do XXVII Simpósio Brasileiro de Informática na Educação (SBIE 2016)

"Estar preparado para utilizar a tecnologia e saber como ela pode dar suporte ao aprendizado são habilidades necessárias no repertório de qualquer profissional docente. Os professores precisam estar preparados para ofertar autonomia a seus alunos com as vantagens que a tecnologia pode trazer. As escolas e as salas de aula, tanto presenciais quanto virtuais, devem ter professores equipados com recursos e habilidades em tecnologia que permitam realmente transmitir o conhecimento ao mesmo tempo que se incorporam conceitos e competências em TIC".

O NMC-HR EES 2014 apresentou um modelo de sala de aula criativa (Creative Classroom - CCR) [Johnson 2014], que contém referência às TICs. CCR é ambiente de aprendizagem inovador que incorpora plenamente o potencial das TIC's para inovar as práticas de ensino-aprendizagem em contextos formais, não-formais e informais. No centro do modelo da CCR situam-se as práticas pedagógicas inovadoras, que surgem quando os professores usam as TIC's nos seus esforços para organizar novas e aperfeiçoadas formas de atividades de aprendizagem abertas, colaborativas e significativas, em vez de simplesmente aumentar as pedagogias tradicionais, como as aulas expositivas e tarefas [Bocconi 2012]. Uma das dimensões do modelo de CCR é o conteúdo e currículo, que contém o parâmetro de referência "atividades significativas". Quando metodologias tradicionais de aprendizagem são empregadas, a tendência é que os estudantes aprendam conceitos e simplesmente reproduzam os métodos para a realização de tarefas apresentados por especialistas. Se o domínio exige também requisitos tais como o estímulo ao raciocínio, as MAA são uma possível solução, fazendo emergir as atividades significativas.

\section{Reflexão}

Diante do exposto, dois aspectos merecem atenção: nas MAA o conhecimento deve ser produzido pelo aluno e a RA é uma tecnologia emergente para visualização de dados que deve estar disponível nos próximos anos. Integrar esses dois pressupostos é interessante e surge um desafio: permitir que estudantes desenvolvam suas capacidades, por meio da produção de conhecimento, fazendo uso de RA e o compartilhem permitindo sua visualização por meio da imersão, interação e envolvimento da comunidade acadêmica na cena real com a inserção de objetos virtuais, aumentando o mundo real e tornando a teoria mais verdadeira e contextualizada. Mas, para incluir as tecnologias de RA em ambientes de MAA algumas questões são preocupantes: 1) como fazer os estudantes, de uma forma geral, e especificamente os de MAA, produzirem seu conhecimento fazendo uso de RA, 2) como tornar a produção de conteúdo de RA tão simples a ponto deste ser produzido por aqueles que não detém conhecimento de computação e 3) como analisar a verdadeira contribuição da RA para CBMAA?

Recentemente, uma revisão sistemática sobre RA na educação médica [Zhu 2014] afirmou que:

- há a necessidade de aprofundar o estudo do uso da RA em educação em saúde uma vez que os estudantes precisam de experiências e nem sempre é possível a interação com objetos reais;

- somente a tecnologia não é suficiente para promover a aprendizagem, mesmo sendo apresentada como um motor para as reformas educacionais;

- novas pesquisas devem ser realizadas na área de educação médica envolvendo RA buscando encontrar o modelo de RA e os projetos educacionais apropriados; 
V Congresso Brasileiro de Informática na Educação (CBIE 2016)

Anais do XXVII Simpósio Brasileiro de Informática na Educação (SBIE 2016)

- antes de afirmar o grande potencial da RA é importante ter uma clara compreensão do seu impacto sobre a aprendizagem.

\section{Materiais e Métodos}

Uma metodologia chamada pesquisa-ação técnica (Technical Action Research - TAR) em junção com o ciclo de engenharia é utilizada no desenvolvimento dessa pesquisa. As atividades de pesquisa a partir dessa junção são: investigação do problema, identificação de artefatos, projeto da solução, validação da solução, implementação da solução e avaliação da implementação. A execução das três primeiras atividades resultaram na escrita desse artigo. Primeiramente, uma busca pelos termos "realidade aumentada" e "educação" em bases científicas da computação e da educação, nacionais e internacionais, foi realizada. Não foram encontrados trabalhos que se assemelham à proposta desse estudo. Somente um trabalho sobre RV, apresentado no SBIE 2003, tem alguma semelhança [Piovesan 2004]. Ao longo de um ano foram realizados encontros, caracterizados por reuniões semanais, com a presença de desenvolvedores, autores desse estudo e professores da medicina e computação da UFSCar, totalizando 109 horas, os quais permitiram a compreensão do processo ensino-aprendizagem de CBMAA. Além dos encontros, os autores também participaram de aulas presenciais em cursos oferecidos pelo IEP para compreensão do processo ensino-aprendizagem por meio da observação. Ao final, um produto de software denominado Sistema de Gestão Pedagógica e Acadêmica (SGPA) para CBMAA foi desenvolvido e foram propostas a abordagem e a arquitetura de software apresentadas na próxima seção. Quanto às tecnologias, o SGPA faz uso do framework Javascript AngularJS no frontend, framework para desenvolvimento web Grails no backend e banco de dados relacional PostgreSQL. Para o ambiente de RA, diversas tecnologias de RA/RV estão sendo analisadas, assim como a possibilidade de desenvolvimento e/ou de incorporação de tecnologias já existentes. Como tecnologias de desenvolvimento encontram-se: Unity 3D, ARToolkit, C\#, Kit de desenvolvimento Kinect e Vuforia. Caso a opção seja pela incorporação de tecnologias os exemplos são: BioDigital Human (biblioteca virtual 3D do corpo humano), Sketchfab (site para compartilhamento de modelos 3D), imagens e animação oferecidas pela empresa 3D4Medical e Project Esper (projeto de RV e RA da empresa 3D4Medical a ser lançado a partir de 2017).

\section{Abordagem em Realidade Aumentada e Arquitetura de Software}

Considerando todas as argumentações até aqui apresentadas são propostas ABORA para CBMAA e uma arquitetura de software para dar suporte à abordagem (figura 3). A partir de estudos do processo ensino-aprendizagem na UFSCar e IEP aspectos como pessoas, seus papéis, processos, estruturas, estratégias, técnicas e resultados foram identificados e compõem a abordagem. A abordagem deve assegurar que estudantes, sem conhecimento prévio de computação, sejam capazes de produzir conteúdo de RA sobre os mais variados domínios, incorporar tal conteúdo ao seu portfolio reflexivo e visualizá-lo em contexto real durante o compartilhamento do conhecimento pemitindo a imersão, interação e envolvimento da comunidade acadêmica por meio de objetos virtuais, que são modelos computacionais previamente preparados e armazenados em um banco de dados, inseridos na cena real, consolidando o processo ensinoaprendizagem de CBMAA. ABORA está baseada no SGPA para CBMAA [Santos 
V Congresso Brasileiro de Informática na Educação (CBIE 2016)

Anais do XXVII Simpósio Brasileiro de Informática na Educação (SBIE 2016)

2016], no emprego de tecnologias de RA e de computação gráfica associadas à Computação Ubíqua e numa biblioteca de imagens para cada domínio do conhecimento, para dar suporte à aquisição e à produção de conhecimento por parte da comunidade acadêmica de CBMAA. Para tanto, faz-se necessário uma arquitetura de software para produção e visualização do conhecimento e posterior avaliação da abordagem.

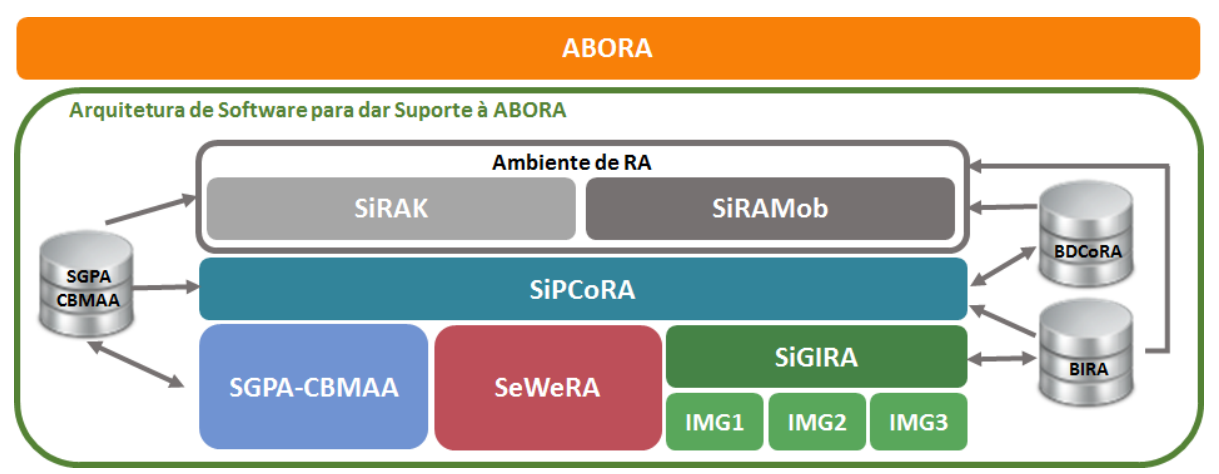

Figura 3. Arquite tura de Software de Apoio à ABORA

\subsection{Abordagem em Realidade Aumentada (ABORA)}

Sabendo-se que sistemas de RA enriquecem o ambiente real com objetos virtuais, possibilitam a imersão e interação dos atores com objetos virtuais inseridos no ambiente real, favorecendo o alcance das competências exigidas em CBMAA, proporcionando uma aproximação entre teoria e realidade, foram selecionados quinze fundamentos para composição da abordagem (tabela 2). Tais fundamentos são a base para a implementação da arquitetura de software, avaliação da abordagem e estudo do impacto de RA sobre o processo ensino-aprendizagem de CBMAA. A escolha desses fundamentos foi baseada no estudo dos ambientes que aplicam MAA, nas tecnologias de hardware e software existentes atualmente e nos estudos apresentados pelo NMC e por Zhu (2014).

Tabela 2. ABORA

\begin{tabular}{|l|l|}
\hline Fundamento & Descrição \\
\hline Visão Geral da Abordagem & Uso de RA em CBMAA \\
\hline Teoria de Aprendizagem & Problem Based Learning ou Aprendizagem Baseada em Problema \\
\hline Estratégia de Aprendizagem & AAD \\
\hline Papel da RA & $\begin{array}{l}\text { Enriquecer o ambiente real com a inserção de objetos virtuais (imagens, videos, } \\
\text { sons e textos) gerados pelo computador, fazendo uso de TIC's de baixo custo }\end{array}$ \\
\hline Objetivo da Abordagem & Provar que RA contribui com o processo ensino-aprendizagem de CBMAA \\
\hline Como? (Metodológico) & $\begin{array}{l}\text { A partir de histórias previamente criadas pelo estudante, que reflitam a criticidade e } \\
\text { competência adquirida por este durante o processo ensino-aprendizagem }\end{array}$ \\
\hline Como? (Tecnológico) & $\begin{array}{l}\text { Estudantes sem conhecimento prévio de computação são capazes de demonstrar seu } \\
\text { aprendizado, diante de situação problema, e fazendo uso de RA como tecnologia de } \\
\text { visualização. Isto ocorre através da criação de cenário com objetos virtuais } \\
\text { sobrepostos sobre a imagem real, previamente preparado e armazenado em um } \\
\text { banco de dados. A RA é usada como tecnologia de visualização para apresentar o } \\
\text { cenário e consolidar o conhecimento adquirido pelo estudante. O cenário criado } \\
\text { permite a imersão, interação e envolvimento da comunidade acadêmica em um } \\
\text { contexto real para compartilhar o conhecimento produzido com outros atores, } \\
\text { consolidando o processo ensino-aprendizagem em CBMAA. }\end{array}$ \\
\hline
\end{tabular}


V Congresso Brasileiro de Informática na Educação (CBIE 2016)

Anais do XXVII Simpósio Brasileiro de Informática na Educação (SBIE 2016)

\begin{tabular}{|c|c|}
\hline Atores & Gestor de Aprendizagem, legislador, especialista, facilitador, professor, estudante \\
\hline Pré Requisito & SGPA-CBMAA, biblioteca de imagens de RA \\
\hline $\begin{array}{l}\text { Tecnologia de Hardware } \\
\text { Empregada }\end{array}$ & $\begin{array}{l}\text { Laptop, computador, servidor, smartphone, tablet, internet móvel, kinect, } \\
\text { Datashow, vídeo, Televisão }\end{array}$ \\
\hline $\begin{array}{l}\text { Tecnologia de Software } \\
\text { Empregada }\end{array}$ & $\begin{array}{l}\text { RA, Computação Gráfica, Computação Ubíqua, Grails, Java, Angular, Javascript, } \\
\text { PostgreSQL, ARToolkit, C\#, Visual Studio, Unity 3D, Biblioteca Kinect, Vuforia }\end{array}$ \\
\hline Eficiência de Custo & $\begin{array}{l}\text { A tecnologia de hardware empregada tem custo acessível à comunidade de } \\
\text { CBMAA pois faz uso de equipamentos de baixo custo ao alcance da população e } \\
\text { das instituições de ensino }\end{array}$ \\
\hline Resultados Esperados & $\begin{array}{l}\text { Estilo próprio de estudo, descoberta individualizada do momento da aprendizagem, } \\
\text { construção do próprio conhecimento, fortalecimento das MAA, fortalecimento do } \\
\text { processo ensino-aprendizagem, popularização da RA. Estas são hipóteses que serão } \\
\text { confirmadas após avaliação da tecnologia de software pela comunidade acadêmica }\end{array}$ \\
\hline Aceitação do Usuário & $\begin{array}{l}\text { Sendo a comunidade acadêmica formada em sua maioria por jovens que já usam a } \\
\text { smartphone e Kinect, a aceitação pelo usuário é alcançada. Hipótese que será } \\
\text { confirmada após avaliação da tecnologia de software pela comunidade acadêmica }\end{array}$ \\
\hline $\begin{array}{l}\text { Impacto da RA sobre o } \\
\text { Processo } \\
\text { Aprendizagem }\end{array}$ & $\begin{array}{l}\text { AAD realizada em qualquer lugar, por qualquer quantidade de alunos, } \\
\text { independentemente de laboratórios e equipamentos específicos, popularização da } \\
\text { RA em CBMAA, uso de novas técnicas de visualização de dados. Estas são } \\
\text { hipóteses que serão confirmadas após avaliação da tecnologia de software pela } \\
\text { comunidade acadêmica }\end{array}$ \\
\hline
\end{tabular}

\subsection{Arquitetura de Software}

Para validar e avaliar a ABORA alguns elementos de software devem ser desenvolvidos. Esses elementos pertencem às categorias de bancos de dados, serviços web, sistema de informação para gerenciamento de conteúdo (CMS - Content Management System) e sistema de RA, descritos a seguir.

Primeiramente, o modelo computacional, formado pelas imagens de RA, deve ser criado. É por meio do CMS SiGIRA (Sistema de Gerenciamento de Imagens de RA) que cada imagem é catalogada, indexada e armazenada no BIRA (Banco de Imagens de RA). Para que os elementos de software que dão suporte à ABORA funcionem, é necessário que exista um SGPA. O SGPA deve ser estendido, dando surgimento ao SiPCoRA (Sistema para Produção de Conhecimento usando RA). O SiPCoRA tem o objetivo de armazenar o conhecimento produzido pelo estudante durante a realização de AAD para as ações educacionais, o que significa encontrar imagens de RA, associá-las à ação educacional, articular histórias dialogando com os problemas narrados ao estudante, formular hipóteses, enumerar e priorizar as ações apresentadas enfim, fundamentar seu conhecimento usando RA. Para facilitar a implementação do SiPCoRA são utilizados serviços web e API's (Application Programming Interface), disponibilizados sob a forma de um conjunto de classes e serviços denominado SeWeRA (Serviço Web para RA). O conhecimento produzido pelo estudante é armazenado no BDCoRA (Banco de Dados de Conhecimento em RA). A partir desse momento o estudante pode, então, fazer uso do ambiente de RA para visualizar seu conhecimento produzido, usando qualquer dos sistemas de RA: SiRAK é ou SiRAMob. O SiRAK (Sistema de RA para Visualização de Conhecimento usando Kinect) permite a visualização do conhecimento produzido pelo estudante em um ambiente cuja infraestrutura prevê o uso de Kinect, computador, câmera e televisão. O estudante seleciona o conhecimento que deseja visualizar, o qual é formado por imagens de RA e 
V Congresso Brasileiro de Informática na Educação (CBIE 2016)

Anais do XXVII Simpósio Brasileiro de Informática na Educação (SBIE 2016)

anotações, previamente elaborado por ele e inserido no BDCoRA. O conteúdo de RA é gerado, objetos virtuais, que compõem o cenário de conhecimento criado pelo estudante, são inseridos no ambiente real, o estudante interage com os objetos virtuais, compartilha seu conhecimento, contextualiza com imagens virtuais bastante próximas da realidade e encerra um ciclo do processo enriquecendo o ambiente ensino-aprendizagem. $O$ SiRAMob (Sistema de RA para Visualização de Conhecimento usando Dispositivo Móvel) tem a mesma finalidade do SiRAK, mas usa uma tecnologia com um menor custo: o smartphone do estudante.

\section{Conclusão e Trabalhos Futuros}

Este artigo apresentou uma abordagem em RA para CBMAA, denominada ABORA, e uma arquitetura de software, para dar apoio à abordagem no sentido de avaliá-la diante da comunidade acadêmica. Para propor a ABORA, estudos sobre o processo ensinoaprendizagem, estratégias de ensino, resultados esperados, pessoas envolvidas, estruturas e técnicas de CBMAA na UFSCar e no IEP foram realizados. Foi proposta uma abordagem formada por 15 fundamentos para definir o escopo da RA no processo ensino-aprendizagem, definindo um modelo de RA adequado ao processo. Além da abordagem, este artigo apresentou uma arquitetura de software para dar apoio à ABORA, a qual é formada por oito elementos de software que vão desde a gestão acadêmica e pedagógica até a visualização de cenários com RA, cenários estes criados pelo estudante e que demonstram seu conhecimento adquirido. A implementação de toda a arquitetura proposta permitirá o uso da RA pela comunidade acadêmica durante momentos de AAD. Por enquanto, somente o componente SGPA está implementado, pesquisa envolvendo a linguagem de programação ARToolkit foi realizada e os estudos estão concentrados no uso da tecnologia Kinect e da plataforma Vuforia para desenvolvimento do ambiente de RA, e também na incorporação de tecnologias já consolidadas de RV e RA na arquitetura de software proposta. Além da parceria com o Departamento de Medicina da UFSCar, há uma parceria com professores do Curso de Física do IFSP para uso do protótipo no ensino de nanociência e nanotecnologia, quando então serão usadas as metodologias TAM (Tecnology Acceptance Model), por meio da escala de Likert, e SAM (Self Assessment Manikin) para avaliação da ABORA e da arquitetura de software. Um estudo sobre padrões abertos para RA, assim como a captação de recursos para desenvolvimento de produtos nacionais, tanto de software como de hardware, necessários para a implementação das tecnologias de software aqui propostas, são contribuições futuras para este estudo.

\section{Referências Bibliográficas}

Bocconi, S. et al (2012). "Innovating Learning: Key Elements for Developing Creative Classrooms", In: European Commission, Joint Research Centre-Institute for Prospective Technological Studies, Seville, Spain, Europe.

Braga, M. (2001). Realidade virtual e educação. Revista de Biologia e Ciências da Terra, Paraíba, v. 1, n. 1, jan/jun.

Johnson, L. et al (2012). The NMC Horizon Report: 2012 Higher Education Edition. Austin, Texas: The New Media Consortium. Disponível em: $<$ http://www.nmc.org/pdf/2012-horizon-report-HE.pdf>. Acesso em 02/05/2016. 
V Congresso Brasileiro de Informática na Educação (CBIE 2016)

Anais do XXVII Simpósio Brasileiro de Informática na Educação (SBIE 2016)

Johnson, L. et al (2013). NMC Horizon Report: 2013 Higher Education Edition. Austin, Texas: The New Media Consortium. Disponível em <http://www.nmc.org/pdf/2013horizon-report-HE.pdf $>$. Acesso em 02/05/2016.

Johnson L. et al (2014). NMC Horizon Report: 2014 Higher Education Edition. Austin, Texas: The New Media Consortium. Disponível em $<$ http://cdn.nmc.org/media/2014nmc-horizon-report-he-EN-SC.pdf> . Acesso em 02/05/2016.

Johnson L. et al (2015). NMC Horizon Report: 2015 Higher Education Edition. Austin, Texas: The New Media Consortium. Disponível em $<$ http://cdn.nmc.org/media/2015nmc-horizon-report-HE-EN.pdf $>$. Acesso em 02/05/2016.

Johnson, L. et al (2016). NMC Horizon Report: 2016 Higher Education Edition. Austin, Texas: The New Media Consortium. Disponível em <http://cdn.nmc.org/media/2016nmc-horizon-report-he-EN.pdf>. Acesso em 02/05/2016.

NMC (2016) - New Media Consortium - Disponível em: <http://www.nmc.org/about>, Acesso em 02/05/2016.

Owen, M. et al (2011). "Pedagogic Issues and Questions from the Science Center to Go, Augmented Reality, Project Implementation". EDEN - Open Classroom Conference. Proceedings of the Science Center To Go Workshops. October, 27-29. p. 13-30. Ellinogermaniki Agogi, Athens, Greece.

Piovesan, A. P. et al. (2004). "ConstruiRV - Uma Ferramenta Educacional para Construir Conhecimento Utilizando Realidade Virtual”, Anais do XIV Simpósio Brasileiro de Informática na Educação. Disponível em <http://www.brie.org/pub/index.php/sbie/article/view/294/280>. Acesso em: 29/05/2016.

Ribeiro, A. C. et al. (200?). Caderno do Curso de Medicina da UFSCar, Universidade Federal de São Carlos.

Santana, E. F. Z. et al (2009). "Um Ambiente Baseado na WEB 2.0 para Atividades de Simulação na Educação Médica Construtivista”, Anais do XX Simpósio Brasileiro de Informática na Educação. Disponível em<http://www.br-ie.org/pub/index.php/ sbie/article/view/1130/1033>. Acesso em: 29/05/2016.

Santos, H. F. et al (2016). "Augmented Reality Approach for Knowledge Visualization and Production (ARAKVP) in Educational and Academic Management System for Courses Based on Active Learning Methodologies (EAMS-CBALM)". In: Information Technology: New Generations. $13^{\text {th }}$ International Conference on Information Technology. Springer. p. 1113-1124. Edited by Shahram Latifi. Las Vegas, Nevada, USA.

Tempski, P. et al (2014). Caderno do Curso de Educação na Saúde para Preceptores do SUS, Instituto Sírio Libanês de Ensino e Pesquisa.

Unesco (2009). "Padrões de Competência em TIC para Professores: Módulos de Padrão de Competência”. Disponível em <http://unesdoc.unesco.org/images/0015/001562/ 156207por.pdf $>$. Acesso em 01/05/2016.

Zhu, E. et al (2014). "Augmented Reality in Healthcare Education: An Integrative Review". PeerJ, 2, e469. Disponível em < https://peerj.com/articles/469/>. Acesso em: 29/05/2016. 